\title{
Feeding habits of Pagellus acarne (Sparidae) in the Gulf of Tunis, central Mediterranean
}

\author{
RAFIKA FEHRI-BEDOUI, EMNA MOKRANI and OUM KALTHOUM BEN HASSINE \\ Unité de Biologie, Ecologie et Parasitologie des Organismes Aquatiques, Faculté des Sciences de Tunis, \\ Campus Universitaire, 2060El Manar II, Tunisie. E-mail: rafika_fr2000@yahoo.fr
}

\begin{abstract}
SUMMARY: The feeding habits of the axillary seabream, Pagellus acarne (Risso, 1810), from the Gulf of Tunis were investigated in relation to season, sex and fish size (juveniles TL $<14.5 \mathrm{~cm}$ and adults $T L \geq 14.5 \mathrm{~cm}$ ). A total of 536 specimens (males, females, unsexed and hermaphroditic), ranging between 11.0 and $25.2 \mathrm{~cm} \mathrm{TL}$, were collected with trammel nets from June 2005 to July 2006. Their stomach contents were analysed. Of the total number of examined stomachs, 279 stomachs were empty (Vacuity index, $V I=52 \%$ ). The $V I$ did not reveal significant seasonal differences when all of the specimens were analysed together; however, significant seasonal variations were observed for females. The diet of the axillary seabream was composed of 36 different prey species. The most important prey were Arthropoda, Mollusca and Echinodermata. Both the dietary indices and the Spearman's coefficient of correlation indicated seasonal variations in the diet. The diet was more diversified in adults than in juveniles. The axillary seabream fed on a wide range of prey items, endofauna and nekton, and can be considered a carnivorous and euryphagous predator.
\end{abstract}

Keywords: Pagellus acarne, diet, dietary indices, Gulf of Tunis, central Mediterranean.

\begin{abstract}
ReSumen: La dieta de Pagellus acarne (Sparidae) en el golfo de Túnez, Mediterráneo central. - Se estudió la dieta del aligote, Pagellus acarne (Risso, 1810), del golfo de Túnez en función de las estación del año, del sexo y de dos grupos de talla: juveniles $(\mathrm{LT}<14.5 \mathrm{~cm}$ ) y adultos $(\mathrm{LT} \geq 14.5 \mathrm{~cm}$ ). Entre junio 2005 y julio 2006 se recolectaron con trasmallos, un total de 536 ejemplares (machos, hembras, sexos no determinados) cuya longitud total oscilaba entre 11 y $25.5 \mathrm{~cm}$. Se analizó el contenido estomacal; 279 estaban vacíos (índice de vacuidad, VI $=52.0 \%$ ). Este índice no reveló diferencias significativas en relación a la estación del año. Sin embargo, se observaron importantes variaciones estacionales para las hembras. Se identificaron un total de 36 especies de presas en la dieta. Las más frecuentes eran artrópodos, moluscos y equinodermos. Los índices de alimentación calculados, así como la aplicación del coeficiente de correlación de Spearman, mostraron variaciones estacionales. El análisis específico de las presas evidenció que la dieta de los machos era diferente a la de las hembras y era más variada en los adultos que en los juveniles. P. acarne se alimenta de un amplio espectro de presas, endofauna y necton, y podría considerarse como depredador carnívoro y eurífago.
\end{abstract}

Palabras clave: Pagellus acarne, dieta, índices de alimentación, golfo de Túnez, Mediterráneo central.

\section{INTRODUCTION}

In the Mediterranean there are 23 Sparidae species, 4 of which belong to the Pagellus genus: the common pandora, Pagellus erythrinus, (Linnaeus, 1758), the axillary seabream, P. acarne (Risso, 1810), the red pandora, $P$. bellottii (Steindachner, 1882 ) and the blackspot seabream $P$. bogaraveo (Brünnich, 1756). The last species is more com- mon on the continental slope, while the other species prefer to inhabit the continental shelf (Bonnet, 1969; Spedicato et al., 2002). Andaloro and Rinaldi (1998) suggested that the presence of $P$. acarne in the Mediterranean is the result of the "tropicalisation" of this sea. The Pagellus species are generally known as hermaphroditic, $P$. acarne and P. bogaraveo as proterandric and $P$. erythrinus and $P$. bellottii as protogynous (D’Ancona, 1949; Larrañeta, 1964; 
Lissia and Frau, 1968; Fischer et al., 1987).

$P$. bellottii has never been recorded in Tunisia; $P$. bogaraveo is confined to the northern coasts of Tunisia whereas $P$. erythrinus and $P$. acarne are found everywhere (Bradaï, 2000). The main target of local fisheries is the common pandora (2441 tons/year, from coastal and trawl fleets). Conversely, landings of the axillary seabream are rather low along the northern coasts and only mentioned occasionally (Lubet and Azouz, 1969; Bouhlel, 1978; Bradaï, 2000). Only one study is available on the reproduction of this species in the Gulf of Tunis (Mokrani et al., 2007). However, the economic interest of the axillary seabream has stimulated some biological studies in other Mediterranean regions, such as in the Ionian Sea (Andaloro, 1983a), Greek waters (Mytilineou, 2000), the Algerian sea (Zerouali-Khodja and Amalou, 2005), around Canary isles (Pajuelo and Lorenzo, 2000) and south Portugal (Coelho et al., 2005). Since 1990, following the adoption of the strategic necessity to diversify the artificial production of finfish species in the Mediterranean (Abellán and Basurco, 1999), the culture of $P$. acarne started in Italy (Greco et al., 1995; Arculeo et al., 2000) and in Spain (Dominguez, 2000). The Feeding habits of $P$. acarne have been studied in Atlantic (Domanevskaya and Patokina, 1984; Morato et al., 2001) and Mediterranean waters (Andaloro, 1983b; Rizkalla et al., 1999).

To improve the stock management of the axillary seabream and to ensure its sustainability, the key parameters relating to its dynamics and exploitation have been defined. Natural and fishing mortalities have been estimated in Atlantic waters (Pajuelo and Lorenzo, 2000). Selectivity of the fishing gears has been studied for this species in Greek (Petrakis and Stergiou, 1996; Tokaç et al., 1998) and Portuguese waters (Santos et al. 1995; Campos and Fonseca, 2003).

The purpose of this work was to study the feeding habits of the axillary seabream in the Gulf of Tunis through stomach content analysis.

\section{MATERIAL AND METHODS}

\section{Fish sampling}

Samples of axillary seabream were collected twice per month from the artisanal fleet operating in the Gulf of Tunis (Fig. 1) with trammel nets of $48 \mathrm{~mm}$ mesh size (stretched). The nets were set at

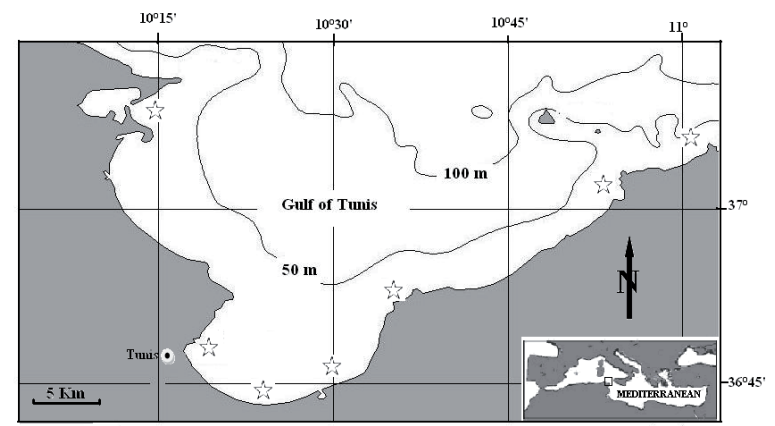

FIG. 1. - Map of the Gulf of Tunis showing the study area of $P$. acarne. (노): Sampling localities.

night and hauled in the morning.

From June 2005 to July 2006, all the axillary seabream specimens caught were collected and considered for this study. Each specimen was measured to the nearest $\mathrm{mm}$ (TL, total length), dissected and sexed. The individuals were classified into two size groups, juveniles $(\mathrm{TL}<14.5 \mathrm{~cm})$ and adults $(\mathrm{TL} \geq 14.5 \mathrm{~cm})$, on the basis of the smallest size at maturity (Mokrani et al., 2007). The stomach was removed from each specimen and preserved in a $70^{\circ}$ alcohol solution.

\section{Stomach content analysis}

The stomach contents were washed in a Petri dish and examined under a microscope. The food items were sorted into large taxonomic groups and, when possible, identified to the species level according to Riedel (1983) and Fischer et al. (1987). When the state of digestion was not advanced, the number of prey was counted. Otherwise, the number of prey was determined from the hard structures. The vacuity index $(V I, \%)$, the frequency of occurrence $(F O$, $\%)$ and the numerical percentage of a prey item i $(N$, $\%)$ were calculated according to Hureau (1970) and Berg (1979) as follows:

$V I=\frac{N v}{N e} \times 100 \quad F O=\frac{N d i}{N n v} \times 100 \quad N=\frac{N t i}{N i} \times 100$ where $N v=$ number of empty stomachs, $N e=$ total number of examined stomachs, $N d i=$ number of stomachs containing the prey i, Nnv = total number of stomachs containing some food, Nti = total number of prey i, Ni= total number of ingested prey.

The point method proposed by Hynes (1950), Berg (1979), Pasquaud et al. (2004), and Bouchereau et al. (2006) was used to estimate the ingested biomass of each item. Each prey is assigned a number of points $(P, \%)$ proportional to its estimated contribution to the whole stomach volume (Table1), as 
TABle 1. - Points given to food items of the diet of $P$. acarne in relation to their digestibility and size according to Pasquaud et al. (2004) and Bouchereau et al. (2006).

\begin{tabular}{lrlr}
\hline Taxa & Points & Taxa & Points \\
\hline Teleostei & 100 & Decapoda (crabs) & 10 \\
Annelida & 40 & Mollusca & 1 \\
Echinodermata & 25 & Bacillariophyta & 1 \\
Decapoda (shrimps) & 25 & Phytophyta & 1 \\
Amphipoda & 10 & Bryozoa & 1 \\
Isopoda & 10 & Nemathelminths & 1 \\
Cumacea & 10 & Foraminifera & 0.5 \\
Mysidacea & 5 & Ostracoda & 0.5 \\
& & & \\
\hline
\end{tabular}

follows:

$$
P=\frac{N p i}{N t p} \times 100
$$

where $N p i=$ total number of points of a prey item, $N t p=$ total number of points.

The food preference of axillary seabream was determined by applying the Main Food Index (MFI) (Zander, 1982):

$$
M F I i=\frac{\sqrt{P i(N i+F i) / 2}}{\sum_{i} M F I i} \times 100
$$

Prey were classified based on $M F I$ values as follows:

MFI $>75=$ preferential prey, $50<M F I<75=$ main prey, $25<M F I<50=$ secondary prey and $M F I$ $<25=$ accessory prey.

Statistical differences of the different indices (VI; $F O ; N)$ in relation to season or sex were assessed by the chi-square goodness of fit test $\left(\chi^{2}\right)$ at $p=0.05$.

Variations in the diet of the axillary seabream were estimated using the Spearman coefficient of correlation $(\rho)$ (Falissard, 2005):

$$
\rho=1-\frac{6 \sum d^{2}}{n^{3}-n}
$$

where $\mathrm{n}=$ rank number and $\mathrm{d}=$ difference between ranks.

The items were classified according to a decreasing order of the coefficient value. For the same sample, the rank attributed to the equally placed items corresponds to the mean ranks that the items would have had if they had not been identical values of the dietary index. Statistical significance of $\rho$ was tested using the t-Student test at $\mathrm{p}=0.01$ :

$$
t=\frac{\rho}{\left(1-\rho^{2}\right)^{1 / 2}} \times(n-2)^{1 / 2}
$$

\section{RESULTS}

A total of 536 specimens of $P$. acarne ranging from $11 \mathrm{~cm}$ to $25.2 \mathrm{~cm}$ were collected over the entire year (Table 2).

\section{Feeding intensity}

Out of 536 stomachs examined, 273 were empty (vacuity index, $V I=52 \%$ ). The vacuity index did not reveal any significant differences over the year $\left(\chi^{2}=3.09 ; \mathrm{p}>0.05\right)$, and accounted for $58 \%$ in summer, $48 \%$ in autumn, $49 \%$ in winter and $53 \%$ in spring. However, the vacuity index computed by sex showed significant seasonal variations for females $\left(V I=51 \% ; \chi^{2}=10.4, \mathrm{p}<0.05\right)$, but did not present significant seasonal variations for males $(V I=52 \%$; $\left.\chi^{2}=1.10, \mathrm{p}>0.05\right)$. VI values only differed significantly between sexes in autumn (Table 3 ). The mean annual $V I$ recorded in juveniles $(\mathrm{Lt}<14.5 \mathrm{~cm})$ was $87.8 \%$ with a maximum in autumn and spring $(100 \%)$ and was higher than that of the adults $(\mathrm{Lt} \geq 14.5 \mathrm{~cm})$

TABLE 2. $-P$. acarne specimens collected in the Gulf of Tunis from June 2005 to July 2006.

\begin{tabular}{lccccc}
\hline Seasons & Females & Males & Unsexed & Hermaphroditics & Total \\
\hline Summer & 54 & 57 & 9 & 1 & 121 \\
Autumn & 34 & 90 & 0 & 0 & 124 \\
Winter & 57 & 103 & 16 & 4 & 180 \\
Spring & 42 & 55 & 11 & 3 & 111 \\
Total & 187 & 305 & 36 & 8 & 536 \\
\hline
\end{tabular}

TABLE 3. - Seasonal variations of the vacuity index $(V I)$ of $P$. acarne and statistical comparison between males and females. SD: Standard deviation.

\begin{tabular}{lcccr}
\hline & \multicolumn{2}{c}{$V I(\%)$} & SD & $\begin{array}{r}\text { Significance } \\
(\mathrm{p}=0.01)\end{array}$ \\
Seasons & Males & Females & & \\
\hline Summer & 53.1 & 65.6 & 0.08 & - \\
Autumn & 59.4 & 32.2 & 0.10 & + \\
Winter & 48.6 & 53.1 & 0.07 & - \\
Spring & 47.4 & 53.3 & 0.09 & - \\
Annual VI $(\%)$ & 52.1 & 51 & 0.04 & - \\
& & & & \\
\hline
\end{tabular}

TABLE 4. - Seasonal variations of the vacuity index $(V I, \%)$ of $P$. acarne in juveniles and adults.

\begin{tabular}{lcc}
\hline Seasons & Juveniles $(\mathrm{TL}<14.5 \mathrm{~cm})$ & Adults $(\mathrm{TL} \geq 14.5 \mathrm{~cm})$ \\
\hline Summer & 64.3 & 55.5 \\
Autumn & 100 & 46.6 \\
Winter & 86.9 & 43.6 \\
Spring & 100 & 52.3 \\
Annual $V I(\%)$ & 87.8 & 49.5 \\
\hline
\end{tabular}


670 • R. FEHRI-BEDOUI et al.

TABle 5. - Prey identified in the stomach contents of $P$. acarne in relation to season. $F O$, frequency of occurrence; $N$, numerical percentage; $P$, point percentage; $M F I$, main food index; R, classification of prey.

\begin{tabular}{|c|c|c|c|c|c|c|c|c|c|c|}
\hline Taxa & $\begin{array}{l}\text { Summer } \\
F O(\%)\end{array}$ & $N(\%)$ & $P(\%)$ & $M F I$ & $\mathrm{R}$ & $\begin{array}{l}\text { Autumn } \\
F O(\%)\end{array}$ & $N(\%)$ & $P(\%)$ & $M F I$ & $\mathrm{R}$ \\
\hline \multicolumn{11}{|l|}{$\begin{array}{l}\text { ARTHROPODA } \\
\text { CRUSTACEA DECAPODA }\end{array}$} \\
\hline Crangon crangon & 1.8 & 1.4 & 1.2 & 1.18 & & 7.8 & 3.9 & 13.5 & 8.9 & \\
\hline Ebalia sp. & 1.8 & 1.2 & 1.3 & 1.4 & & 11 & 4.6 & 5.9 & 6.7 & \\
\hline Liocarcinus arcuatus & 1.8 & 0.4 & 0.7 & 0.8 & & - & - & - & - & \\
\hline Pagurus sp. & 3.7 & 2.6 & 2.1 & 2.3 & & 1.6 & 0.3 & 0.4 & 0.6 & \\
\hline Unidentified Decapoda & - & - & - & - & - & - & - & - & - & \\
\hline Total Decapoda & 9.2 & 3.5 & 5.3 & 5.7 & & 20.3 & 8.8 & 19.8 & 16.2 & \\
\hline MYSIDA & & & & & & & & & & \\
\hline $\begin{array}{l}\text { Mysis sp. } \\
\text { ISOPODA }\end{array}$ & 14.8 & 10.1 & 6.9 & 9.2 & & 26.6 & 9.7 & 6.3 & 10.7 & \\
\hline Sphaeroma serratum & 9.2 & 12.1 & 18.1 & 13.9 & & 9.4 & 4.8 & 6.3 & 6.7 & \\
\hline CUMACEA & - & - & - & - & & 1.6 & 1 & 1 & 1.2 & \\
\hline OSTRACODA & & & & & & & & & & \\
\hline $\begin{array}{l}\text { Cypridina mediterranea } \\
\text { AMPHIPODA }\end{array}$ & 3.7 & 1.2 & 0.1 & 0.4 & & 10.9 & 2.6 & 0.1 & 1 & \\
\hline Gammarus gammarus & 13 & 7. & 9.8 & 9.9 & & 1.6 & 1 & 1.2 & 1.2 & \\
\hline Total Arthropoda & 50.2 & 37.9 & 40.1 & 39.1 & (1) & 70.2 & 27.9 & 35 & 37.1 & (2) \\
\hline \multicolumn{11}{|l|}{ MOLLUSCA } \\
\hline Flexopecten flexиosus & 7.7 & 0.7 & 0.4 & 2.1 & & - & - & - & - & \\
\hline Unidentified & 41.4 & 25.9 & 3.5 & 10.3 & & 34.6 & 14.5 & 1.7 & 7.3 & \\
\hline \multicolumn{11}{|l|}{ GASTEROPODA } \\
\hline Phyllonotus trunculus & - & - & - & - & & - & - & - & - & \\
\hline Calliostoma granulatum & 8.56 & 5.72 & 0.31 & 1.43 & & 4.68 & 3.92 & 0.35 & 1.08 & \\
\hline Calliostoma ziziphinum & - & - & - & - & & - & - & - & - & \\
\hline Cerithium alucastrum & 0.14 & 0.01 & 0.01 & 0.02 & & - & - & - & - & \\
\hline Lunatia sp. & 4.4 & 0.8 & 0.1 & 0.5 & & 6.2 & 0.3 & 0.03 & 0.3 & \\
\hline Turritella communis & 7.4 & 1.6 & 0.4 & 1.2 & & 3.1 & 3.5 & 0.4 & 1. & \\
\hline Caecum trachea & 4.4 & 0.8 & 0.1 & 0.5 & & - & - & - & - & \\
\hline Philine catena & - & - & - & - & & 1.6 & 0.6 & 0.2 & 0.4 & \\
\hline Rissoa sp. & 2.8 & 0.4 & 0.04 & 0.2 & & 3.1 & 1.3 & 0.1 & 0.4 & \\
\hline Retusa sp. & 2.8 & 0.4 & 0.04 & 0.2 & & 9.1 & 1 & 0.4 & 1.2 & \\
\hline SCAPHOPODA & & & & & & & & & & \\
\hline Antalis dentalis & 3.7 & 1.6 & 0.2 & 0.7 & & 4.7 & 2.6 & 0.3 & 1.1 & \\
\hline CEPHALOPODA & & & & & & & & & & \\
\hline Sepia officinalis & - & - & - & - & & 1.6 & 0.3 & 0.04 & 0.2 & \\
\hline Total Mollusca & 83.3 & 37.9 & 5 & 17.1 & (3) & 68.7 & 28.1 & 3.5 & 13 & (3) \\
\hline \multicolumn{11}{|l|}{ ECHINODERMATA } \\
\hline Paracentrotus lividus & - & - & - & - & & 7.8 & 2.3 & 7.5 & 8.1 & \\
\hline Neolampas rostellata & 7.4 & 3.9 & 14.1 & 8.9 & & 21.9 & 8.4 & 34.8 & 22.9 & \\
\hline Amphipholis squamata & 11 & 8.2 & 25.1 & 18.4 & & 28.7 & 7.4 & 29.6 & 19.7 & \\
\hline Total Echinodermata & 18.5 & 12.1 & 39.2 & 27.3 & (2) & 48.4 & 18.1 & 71.8 & 50.7 & (1) \\
\hline \multicolumn{11}{|l|}{ ANNELIDA } \\
\hline Nereis $\mathrm{sp}$ & 7.4 & 2.3 & 10.8 & 6.6 & - & - & - & - & & \\
\hline Sabellidae & 1.8 & 0.8 & 3.9 & 3.5 & - & - & - & - & & \\
\hline Serpulidae & 1.8 & 0.8 & 3.9 & 3.5 & & 4.7 & 1.3 & 6.8 & 4.5 & \\
\hline Total Annelida & 11.1 & 3.9 & 18.7 & 13.6 & (4) & & 1.3 & 6.8 & 4.5 & (4) \\
\hline BACILLARIOPHYTA & 1.8 & 0.8 & 0.1 & 0.3 & (8) & 10.9 & 6.1 & 0.8 & 2.5 & (6) \\
\hline BRYOZOA & 3.7 & 1.9 & 0.2 & 0.8 & (6) & 4.7 & 1.3 & 0.1 & 0.6 & (8) \\
\hline FORAMINIFERA & 9.2 & 5.8 & 0.4 & 1.7 & (5) & 25 & 12.6 & 0.78 & 3.8 & (5) \\
\hline NEMATHELMINTHA & - & - & - & - & & 1.6 & 0.3 & 0.04 & 0.2 & (9) \\
\hline TELEOSTEI & - & - & - & - & & - & - & - & - & \\
\hline \multicolumn{11}{|l|}{ РНYTOPHYТA } \\
\hline Pyrocystis lunula & 3.7 & 1.2 & 0.1 & 0.6 & & 11 & 2.9 & 0.3 & 1.5 & \\
\hline Total Phytophyta & 3.7 & 1.2 & 0.1 & 0.6 & (7) & 10.9 & 2.9 & 0.3 & 1.5 & (7) \\
\hline
\end{tabular}

(Table 4). The VI did not show any significant variations with season for either size group $\left(\chi_{\text {Juveniles }}^{2}=\right.$ $\left.5.92, \mathrm{p}>0.05 ; \chi_{\text {Adults }}^{2}=4.05, \mathrm{p}>0.05\right)$.

\section{Diet composition}

The stomach contents of the axillary seabream were composed of 36 different taxa belonging to nine 
TABle 5 (cont.). - Prey identified in the stomach contents of $P$. acarne in relation to season. $F O$, frequency of occurrence; $N$, numerical percentage; $P$, point percentage; $M F I$, main food index; R, classification of prey.

\begin{tabular}{|c|c|c|c|c|c|c|c|c|c|c|}
\hline Taxa & $\begin{array}{c}\text { Winter } \\
F O(\%)\end{array}$ & $N(\%)$ & $P(\%)$ & $M F I$ & $\mathrm{R}$ & $\begin{array}{l}\text { Spring } \\
F O(\%)\end{array}$ & $N(\%)$ & $P(\%)$ & $M F I$ & $\mathrm{R}$ \\
\hline \multicolumn{11}{|l|}{$\begin{array}{l}\text { ARTHROPODA } \\
\text { CRUSTACEA DECAPODA }\end{array}$} \\
\hline Crangon crangon & 13.4 & 8.1 & 28.7 & 17.4 & & 13.5 & 12.1 & 42.3 & 19 & \\
\hline Ebalia sp. & 10.3 & 5.3 & 5.6 & 5.8 & & 3.8 & 1.3 & 2.1 & 1.9 & \\
\hline Liocarcinus arcuatus & 12.3 & 4 & 1.6 & 2.9 & & 1.9 & 0.7 & 0.3 & 0.5 & \\
\hline Pagurus sp. & 3.3 & 1.5 & 1.6 & 1.8 & & 5.8 & 2 & 2 & 2.3 & \\
\hline Unidentified Decapoda & - & - & - & - & & 1.9 & 0.7 & 0.3 & 0.5 & \\
\hline Total Decapoda & 39.1 & 18.8 & 37.5 & 44.3 & & 26.9 & 27 & 46.9 & 24.1 & \\
\hline MYSIDA & & & & & & & & & & \\
\hline $\begin{array}{l}\text { Mysls sp. } \\
\text { ISOPODA }\end{array}$ & 5.4 & 8.1 & 4.7 & 5.6 & & 3.8 & 2 & 1 & 2.1 & \\
\hline Sphaeroma serratum & 6.5 & 3.3 & 3.8 & 4.3 & & 15.4 & 9.7 & 9.4 & 8 & \\
\hline CUMACEA & 1.1 & 0.5 & 0.8 & 0.8 & & - & - & - & - & \\
\hline OSTRACODA & & & & & & & & & & \\
\hline $\begin{array}{l}\text { Cypridina mediterranea } \\
\text { AMPHIPODA }\end{array}$ & 10.9 & 8.5 & 0.4 & 1.9 & & 1.9 & 1.3 & 0.1 & 0.3 & \\
\hline Gammarus gammarus & 20.1 & 18.1 & 13.9 & 13.3 & & 11.5 & 9.7 & 9.4 & 1 & \\
\hline Total Arthropoda & 83.1 & 57.3 & $\mathbf{5 7 . 3}$ & 70.3 & (1) & 59.6 & 49.8 & 66.7 & 42.4 & (1) \\
\hline \multicolumn{11}{|l|}{ MOLLUSCA } \\
\hline Flexopecten flexuosus & 0.2 & 0.8 & 0.2 & 0.6 & & - & - & - & - & \\
\hline Unidentified & 9.6 & 5.3 & 0.4 & 1.6 & & - & - & - & - & \\
\hline \multicolumn{11}{|l|}{ GASTEROPODA } \\
\hline Phyllonotus trunculus & - & - & - & - & & 14.1 & 7.3 & 1.4 & 4.8 & \\
\hline Calliostoma granulatum & 2.3 & 0.5 & 0.1 & 2.3 & & - & - & - & - & \\
\hline Calliostoma ziziphinum & - & - & - & - & & 5.7 & 2 & 0.2 & 0.7 & \\
\hline Cerithium alucastrum & - & - & - & - & & - & - & - & - & \\
\hline Lunatia sp. & - & - & - & - & & - & - & - & - & \\
\hline Turritella communis & 1.1 & 0.5 & 0.06 & 0.2 & & 7.7 & 2.7 & 0.3 & 0.9 & \\
\hline Caecum trachea & - & - & - & - & & 3.8 & 0.2 & 0.3 & 0.6 & \\
\hline Philine catena & 1.1 & 0.2 & 0.02 & 0.2 & & - & - & - & - & \\
\hline Rissoa sp. & 1.1 & 0.2 & 0.02 & 0.2 & & 1.8 & 0.2 & 0.3 & 0.6 & \\
\hline Retusa sp & 2.2 & 0.5 & 0.1 & 0.2 & & - & - & - & - & \\
\hline SCAPHOPODA & & & & & & & & & & \\
\hline Antalis dentalis & 2.2 & 0.5 & 0.05 & 0.2 & & - & - & - & - & \\
\hline $\begin{array}{l}\text { CEPHALOPODA } \\
\text { Sepia officinalis }\end{array}$ & 1.1 & 0.2 & 0.02 & 0.1 & & _- & _- & - & _ & \\
\hline Total Mollusca & 21 & 8.8 & 0.8 & 3.6 & (5) & 33.2 & 13.5 & 2.3 & 7.8 & (3) \\
\hline \multicolumn{11}{|l|}{ ECHINODERMATA } \\
\hline Paracentrotus lividus & 2.2 & 0.5 & 1.4 & 1.4 & & 3.8 & 1.3 & 3.4 & 2.4 & \\
\hline Neolampas rostellata & 1.1 & 0.2 & 0.7 & 0.7 & & 3.8 & 1.3 & 3.4 & 2.4 & \\
\hline Amphipholis squamata & 12 & 6 & 14 & 10.1 & & 28.8 & 27.8 & 27 & 28.9 & \\
\hline Total Echinodermata & 15.2 & 6.78 & 16.1 & 12.9 & (2) & 36.5 & 30.4 & 33.8 & 33.7 & (2) \\
\hline \multicolumn{11}{|l|}{ ANNELIDA } \\
\hline Nereis $\mathrm{sp}$ & 4.3 & 1.9 & 15.1 & 6.3 & & - & - & - & - & \\
\hline Sabellidae & 4.3 & 2.1 & 6.6 & 5.5 & & - & - & - & - & \\
\hline Serpulidae & - & - & - & - & & - & - & - & - & \\
\hline Total Annelida & 8.7 & 4 & 21.7 & 11.8 & (3) & - & - & - & - & - \\
\hline BACILLARIOPHYTA & 1.1 & 0.2 & 0.02 & 0.1 & (10) & 3.8 & 2 & 0.2 & 0.6 & (6) \\
\hline BRYOZOA & 1.1 & 0.5 & 0.05 & 0.2 & (9) & - & - & - & - & - \\
\hline FORAMINIFERA & 17.4 & 9.8 & 0.5 & 2.7 & (6) & 17.3 & 7.4 & 0.4 & 1.7 & (4) \\
\hline NEMATHELMINTHA & 5.4 & 2.3 & 0.2 & 1 & (8) & - & - & - & - & - \\
\hline TELEOSTEI & 3.3 & 1 & 12.5 & 5.2 & (4) & - & - & - & - & - \\
\hline \multicolumn{11}{|l|}{ PHYTOPHYТА } \\
\hline Pyrocystis lunula & 13.4 & 4 & 0.4 & 1.9 & & 7.7 & 2.7 & 0.3 & 0.9 & \\
\hline Total Phytophyta & 13 & 4 & 0.4 & 1.9 & (7) & 7.7 & 2.7 & 0.3 & 0.9 & (5) \\
\hline
\end{tabular}

major taxa (Table 5). The most important prey were Arthropoda (10 taxa), Mollusca (14 taxa) and Echinodermata (3 taxa). Arthropoda were mainly represented by crustaceans such as Decapoda (Crangon crangon, Ebalia sp., Liocarcinus arcuatus, Pagurus sp.), Amphipoda (Gammarus gammarus), Mysida (Mysis sp.), Isopoda (Sphaeroma serratum), and one unidentified Cumacea and one Ostracoda (Cypridina 
mediterranea). The phylum Mollusca was represented by Gastropoda (Phyllonotus trunculus, Calliostoma granulatum, C. ziziphinum, Cerithium alucastrum, Lunatia sp., Turritella communis, Caecum trachea, Philine catena, Rissoa sp. and Retusa sp.), 2 bivalves (Flexopecten flexuosus and one unidentified), 1 Scaphopoda (Antalis dentalis) and $1 \mathrm{Ce}$ phalopoda (Sepia officinalis). Echinodermata were represented by 1 Echinoideae (Pracentrotus lividus), 1 Neolampapidae (Neolampas rostellata) and 1 Amphiuridae (Amphipholis squamata). It should be noted that both Gastropoda and bivalve shells were found intact in the alimentary tract of the axillary seabream. Annelida were represented by 3 taxa, namely Nereis sp., Serpulidae and Sabellidae. Bacillariophyta, Bryozoa, Foraminifera, Nemathelmintha and Teleostei, were only represented by single taxa. Finally, Phytophyta were exclusively represented by Dinophyceae (Pyrocystis lunula).

\section{Seasonal variations in the diet}

Dietary indices calculated for each prey indicated that the axillary seabream fed mainly on Arthropoda and Mollusca over the year (Table 5). Arthropoda, more frequent and abundant in winter and autumn, showed significant seasonal variations in frequency of occurrence $\left(\chi^{2}=8.64, \mathrm{p}<0.05\right)$ but not in abundance $\left(\chi^{2}=6.29, p>0.05\right)$. The point method showed that Arthropoda were more than $50 \%$ of the ingested biomass in spring $(P=66.7 \%)$, while in summer and autumn they represented only $40.1 \%$ and $35 \%$ respectively, which shows significant seasonal variations $\left(\chi^{2}=17.21, \mathrm{p}<0.05\right)$. Mollusca attained the highest frequency of occurrence in summer $(F O=$ $83.3 \%)$ and the lowest in winter $(F O=21 \%)$, thus revealing significant seasonal variations $\left(\chi^{2}=46\right.$, $\mathrm{p}<0.05)$. Arthropoda constituted the main prey of the axillary seabream in winter $(M F I=70)$ and secondary prey during the rest of the year, while Mollusca were accessory prey all year round $(M F I<25)$. Echinodermata were secondary prey $(13<M F I<51)$, and showed seasonal variations in frequency of occurrence $\left(\chi^{2}=50.77, \mathrm{p}<0.05\right)$, number $\left(\chi^{2}=13.19\right.$, $\mathrm{p}<0.05)$ and biomass $\left(\chi^{2}=69.58, \mathrm{p}<0.05\right)$. Annelida were absent in the diet in spring, poorly represented in autumn and increased in winter and summer. The other prey contributed very little to the ingested biomass (Table 5). The ingestion of fish, Bryozoa and Nemathelmintha was seasonal and scarce. The Spearman coefficient of correlation confirmed the
TABLE 6. - Statistical comparison of the diet of P. acarne according to season. $\rho$, Spearman coefficient of correlation; $t_{\text {obs }}$, Student test values; (+), homogeneous diet; (-), heterogeneous diet.

\begin{tabular}{lccc}
\hline Seasons & $\rho$ & $\mathrm{t}_{\mathrm{obs}}$ & Significance $(\mathrm{p}=0.01)$ \\
\hline Summer-Autumn & 0.936 & 7.521 & + \\
Autumn-Winter & 0.624 & 2.258 & - \\
Winter-Spring & 0.506 & 1.659 & - \\
Spring-Summer & 0.765 & 3.359 & + \\
\hline
\end{tabular}

changes in the diet of the axillary seabream, which indicates seasonal differences in diet. Homogeneity of diet was noted between spring and summer and between summer and autumn (Table 6).

\section{Food in relation to sex}

The diet composition of $P$. acarne varied seasonally in relation to sex, attaining 19 to 22 taxa of prey for females and 14 to 22 taxa for males (Tables 7 and 8). Arthropoda showed significant seasonal variations in the ingested biomass in males $\left(\chi^{2}=91.13\right.$, $\mathrm{p}>0.05)$ but not in females $\left(\chi^{2}=4.38, \mathrm{p}<0.05\right)$. In summer, Mollusca predominated both in number and in frequency of occurrence in both males and females (Tables 7 and 8).

The Mollusca contribution to the ingested biomass, varied between $0.6 \%$ and $4.8 \%$ for males and between $1.2 \%$ and $7.2 \%$ for females and did not show any significant seasonal difference for either $\operatorname{sex}\left(\chi_{\left.(\mathrm{P})^{\circ}\right)}^{2}=6.96, \mathrm{p}>0.05 ; \chi_{(\mathrm{P} P)}^{2}=3.28, \mathrm{p}>0.05\right)$. According to the Main Food Index value, Arthropoda constituted the main prey for both sexes in winter, but became the secondary prey in spring and summer for males and accessory for females (Tables 7 and 8). However, Mollusca were accessory prey for both sexes all year round. Echinodermata were always present in the diet of the axillary seabream, and showed the highest indices in autumn for both sexes. These indices varied significantly during the year

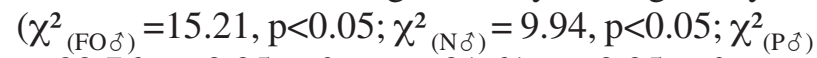
$=29.76, \mathrm{p}<0.05 ; \chi_{(\mathrm{FO})}^{2}=31.61, \mathrm{p}<0.05 ; \chi_{(\mathrm{N} q)}^{2}=$ $\left.12.59, \mathrm{p}<0.05 ; \chi_{(\mathrm{P} q)}^{2}=41.33, \mathrm{p}<0.05\right)$. Echinodermata were the main prey in males and secondary prey in females in autumn but accessory prey in winter for both sexes. The seasonal variations of the ingested prey and differences between male and female diet were confirmed by the Spearman test (Table 9). No significant seasonal variations were observed in females $(0.383<\rho<0.754)$. However, in males, variations were observed between autumn and winter and between autumn and spring. 
TABle 7. - Prey identified in the stomach contents of $P$. acarne females according to season. $F O$, frequency of occurrence; $N$, numerical percentage; $P$, point percentage; $M F I$, Main Food Index; R, classification of prey.

\begin{tabular}{|c|c|c|c|c|c|c|c|c|c|c|}
\hline Таха & $\begin{array}{l}\text { Summer } \\
F O(\%)\end{array}$ & $N(\%)$ & $P(\%)$ & $M F I$ & $\mathrm{R}$ & $\begin{array}{l}\text { Autumn } \\
F O(\%)\end{array}$ & $N(\%)$ & $P(\%)$ & $M F I$ & $\mathrm{R}$ \\
\hline Arthropoda & 80.9 & 33.8 & 51.8 & 46.4 & 1 & 69.1 & 39.6 & 46.9 & 42.7 & 1 \\
\hline Mollusca & 88 & 35.6 & 7.2 & 21 & 2 & 61 & 30 & 2.9 & 11.6 & 3 \\
\hline Echinodermata & 19 & 8.3 & 23.8 & 15.3 & 3 & 47.7 & 17.3 & 38 & 38.6 & 2 \\
\hline Annelida & 19 & 3.1 & 21.8 & 13.8 & 4 & - & - & - & - & - \\
\hline Bacillariophyta & - & - & - & - & - & 4.3 & 2.2 & 1 & 1.1 & 6 \\
\hline Bryozoa & 4.8 & 0.8 & 0.1 & 0.5 & 6.5 & 4.3 & 1.1 & 0.7 & 0.7 & 7 \\
\hline Foraminifera & 14.3 & 4.7 & 0.4 & 1.6 & 5 & 17 & 13.2 & 3.2 & 3.2 & 4 \\
\hline Nemathelmintha & - & - & - & - & - & - & - & - & - & - \\
\hline Teleostei & - & - & - & - & - & - & - & - & - & - \\
\hline Phytophyta & 4.8 & 0.8 & 0.2 & 0.5 & 6.5 & 13 & 3.3 & 2 & 2.1 & 5 \\
\hline Taxa & $\begin{array}{c}\text { Winter } \\
F O(\%)\end{array}$ & $N(\%)$ & $P(\%)$ & $M F I$ & $R$ & $\begin{array}{l}\text { Spring } \\
F O(\%)\end{array}$ & $N(\%)$ & $P(\%)$ & $M F I$ & $R$ \\
\hline Arthropoda & 68.6 & 58.1 & 49.9 & 55.4 & 1 & 71.3 & 30.8 & 45.9 & 36.9 & 1 \\
\hline Mollusca & 20 & 12.6 & 1.2 & 4.7 & 6 & 50.7 & 30.5 & 3.1 & 9.6 & 3 \\
\hline Echinodermata & 16.7 & 5.3 & 14.4 & 14.3 & 2 & 42.8 & 14.8 & 46.5 & 32.4 & 2 \\
\hline Annelidae & 6.7 & 2.7 & 11.3 & 7.7 & 3 & - & - & - & - & - \\
\hline Bacillariophyta & 3.3 & 0.9 & 0.1 & 0.5 & 9.5 & 4.8 & 4.9 & 0.6 & 1.5 & 4 \\
\hline Bryozoa & 3.3 & 0.9 & 0.1 & 0.5 & 9.5 & - & - & - & - & - \\
\hline Foraminifera & 23.3 & 20.5 & 1.1 & 5.1 & 5 & - & - & - & - & - \\
\hline Nemathelminths & 6.7 & 1.8 & 0.2 & 0.9 & 8 & - & - & - & - & - \\
\hline Teleostei & 3.3 & 1.8 & 18.8 & 7.4 & 4 & - & - & - & - & $=$ \\
\hline Phytophyta & 13.3 & 3.6 & 0.4 & 1.8 & 7 & 9.5 & 3.6 & 0.4 & 1.4 & 5 \\
\hline
\end{tabular}

TABLE 8. - Prey identified in the stomach contents of Pagellus acarne males in relation to season. $F O$, frequency of occurrence; $N$, numerical percentage; $P$, point percentage; $M F I$, Main Food Index; R, classification of prey.

\begin{tabular}{|c|c|c|c|c|c|c|c|c|c|c|}
\hline Таха & $\begin{array}{l}\text { Summer } \\
F O(\%)\end{array}$ & $N(\%)$ & $P(\%)$ & $M F I$ & $\mathrm{R}$ & $\begin{array}{l}\text { Autumn } \\
F O(\%)\end{array}$ & $N(\%)$ & $P(\%)$ & $M F I$ & $\mathrm{R}$ \\
\hline Arthropoda & 53.3 & 35 & 33.1 & 38.6 & 1 & 48.7 & 15.9 & 18.1 & 18.6 & 2 \\
\hline Mollusca & 40 & 39.3 & 4.8 & 16.8 & 3 & 36.6 & 16.2 & 3.1 & 7 & 3 \\
\hline Echinodermata & 33.4 & 14.5 & 39.3 & 31.4 & 2 & 56.9 & 36.9 & 73.3 & 58.9 & 1 \\
\hline Annelida & 6.7 & 3.4 & 16.8 & 9.6 & 4 & 4.9 & 0.5 & 4 & 2.8 & 6 \\
\hline Bacillariophyta & - & - & - & - & - & 14.6 & 7.5 & 1.5 & 3.5 & 5 \\
\hline Bryozoa & - & - & - & - & - & 4.5 & 1 & 0.2 & 0.6 & 8 \\
\hline Foraminifera & 3.3 & 0.8 & 0.05 & 0.4 & 6 & 41.5 & 13.4 & 1.3 & 5.2 & 4 \\
\hline Nemathelmintha & - & - & - & - & - & - & - & - & - & - \\
\hline Teleostei & - & - & - & - & - & - & - & - & - & - \\
\hline \multirow[t]{2}{*}{ Phytophyta } & 3.3 & 0.8 & 0.1 & 0.5 & 5 & 17.1 & 4 & 0.8 & 2.5 & 7 \\
\hline & $\begin{array}{l}\text { Winter } \\
F O(\%)\end{array}$ & $N(\%)$ & $P(\%)$ & $M F I$ & $\mathrm{R}$ & $\begin{array}{l}\text { Spring } \\
F O(\%)\end{array}$ & $N(\%)$ & $P(\%)$ & $M F I$ & $\mathrm{R}$ \\
\hline Arthropoda & 76.8 & 45.1 & 49.2 & 50.4 & 1 & 70 & 30.2 & 50 & 45.5 & 1 \\
\hline Mollusca & 18.6 & 4.8 & 0.6 & 2.4 & 5 & 70 & 25.1 & 2.8 & 10.8 & 3 \\
\hline Echinodermata & 21 & 7.5 & 25.1 & 17 & 2 & 43.3 & 16.7 & 45 & 35.3 & 2 \\
\hline Annelida & 12.5 & 9.6 & 23.8 & 12.7 & 3 & - & - & - & - & - \\
\hline Bacillariophyta & - & - & - & - & - & - & - & - & - & - \\
\hline Bryozoa & - & - & - & - & - & - & - & - & - & - \\
\hline Foraminifera & 17.5 & 6.1 & 0.3 & 1.8 & 7 & 26.6 & 10.4 & 0.6 & 3.1 & 4 \\
\hline Nemathelmentha & 5.3 & 2.7 & 0.3 & 1 & 8 & - & - & - & - & - \\
\hline Teleostei & 3.5 & 2.8 & 9.9 & 4.3 & 4 & - & - & - & - & - \\
\hline Phytophyta & 14 & 4.8 & 0.5 & 2 & 6 & 6.7 & 2.1 & 0.2 & 0.9 & 5 \\
\hline
\end{tabular}

\section{Seasonal variations in relation to fish size}

The stomach content analysis of juveniles showed that their diet was poorly diversified in winter, as it consisted of 6 prey taxa, which increased to 10 prey taxa in summer; taxa belonged to Arthropoda, Mollusca and Annelida (Table 10). However, the adult diet was more diversified with 10 prey groups in winter and 8 groups in summer. The most representative prey groups were Arthropoda, Mollusca, Annelida and Echinodermata (Table 11). Ingestion of Arthropoda showed 
TABle 9. - Statistical comparaison of Pagellus acarne diet in relation to sex; $\rho$, Spearman coefficient of correlation; $t_{\text {obs }}$, Student test values; (+), homogeneous diet; (-), heterogeneous diet.

\begin{tabular}{lccc}
\hline Seasons & $\rho$ & $\mathrm{t}_{\mathrm{obs}}$ & Signification $(\mathrm{p}=0.01)$ \\
\hline Females & & & \\
$\quad$ Summer-Autumn & 0.754 & 3.2466 & - \\
Autumn-Winter & 0.418 & 1.301 & - \\
Winter-Spring & 0.383 & 1.172 & - \\
Spring-Summer & 0.617 & 2.217 & + \\
Males & & & - \\
Summer-Autumn & 0.822 & 4.082 & - \\
Autumn-Winter & 0.634 & 2.318 & + \\
Winter-Spring & 0.447 & 1.413 & \\
Spring-Summer & 0.847 & 4.506 & \\
\hline
\end{tabular}

significant variations between summer and winter in frequency of occurrence and number in juveniles $\left(\chi_{(\mathrm{FO})}^{2}=58.50, \mathrm{p}<0.05 ; \chi_{(\mathrm{N})}^{2}=53.80, \mathrm{p}<0.05\right)$ but not in adults $\left(\chi_{(\mathrm{FO})}^{2}=2.965, \mathrm{p}<0.05 ; \chi^{2}{ }_{(\mathrm{N})}=\right.$ $3.78, \mathrm{p}<0.05)$. Their contribution to the ingested biomass, constituted mainly by Amphipoda and Mysida (Mysis sp.) in juveniles and Decapoda in adults, increased according to size in winter and decreased in summer (Tables 10 and 11); there were significant variations between these two seasons only for juveniles $\left(\chi_{(\mathrm{P}, \text { juveniles })}^{2}=23.45\right.$, $\left.\mathrm{p}<0.05 ; \chi_{(\mathrm{P}, \text { adults })}^{2}=0.165, \mathrm{p}>0.05\right)$. According to the MFI, Arthropoda constituted secondary prey for juveniles both in summer and in winter and main prey for adults in summer and secondary prey in winter. The contribution of Mollusca to the ingested biomass in juveniles varied significantly between summer and winter both in frequency of occurrence and in number $\left(\chi_{(\mathrm{FO})}^{2}=54.65, \mathrm{p}<0.05\right.$; $\left.\chi_{(\mathrm{N})}^{2}=90.03, \mathrm{p}<0.05\right)$. This prey group, including mainly bivalve taxa, constituted a secondary prey in summer and an accessory prey in winter. Its presence in the diet of the axillary seabream decreased according to size and it became the accessory prey in adults, both in summer and winter. In juveniles, the ingested biomass of Annelida showed significant variations between summer and winter $\left(\chi_{(\mathrm{P})}^{2}=27.80, \mathrm{p}<0.05\right)$. This biomass decreased according to size chiefly in winter and therefore Annelida were secondary prey in juveniles and accessory prey in adults. Echinodermata, absent in the juvenile diet both in winter and summer, seemed to be appreciated indiscriminately by the adults during these two seasons $\left(\chi_{(\mathrm{F})}^{2}=0.619\right.$, $\mathrm{p}>0.05 ;\left(\chi_{(\mathrm{N})}^{2}=1.368, \mathrm{p}>0.05 ; \chi_{(\mathrm{P})}^{2}=1.74, \mathrm{p}>0.05\right)$. They were secondary prey in summer and accessory prey in winter (Table 11), whereas all the other taxa were accessory prey. The Spearman test indicated differences between the summer and winter diets for both juveniles $\left(\rho=0.945 ; \mathrm{t}_{\mathrm{obs}}=8.17\right.$ $\mathrm{p}<0.01)$ and adults $\left(\rho=0.9 ; \mathrm{t}_{\mathrm{obs}}=5.85 \mathrm{p}<0.01\right)$.

TABLE 10. - Prey identified in the stomach contents of $P$. acarne juveniles in relation to season. $F O$, frequency of occurrence; $N$, numerical percentage; $P$, point percentage; $M F I$, Main Food Index; R, classification of prey.

\begin{tabular}{|c|c|c|c|c|c|c|c|c|c|c|}
\hline Taxa & $\begin{array}{l}\text { Summer } \\
F O(\%)\end{array}$ & $N(\%)$ & $P(\%)$ & $M F I$ & $\mathrm{R}$ & $\begin{array}{l}\text { Winter } \\
F O(\%)\end{array}$ & $N(\%)$ & $P(\%)$ & $M F I$ & $\mathrm{R}$ \\
\hline \multicolumn{11}{|c|}{ ARTHROPODA } \\
\hline \multicolumn{11}{|c|}{ CRUSTACEA DECAPODA } \\
\hline $\begin{array}{l}\text { Pagurus sp. } \\
\text { MYSIDA }\end{array}$ & 7.1 & 1.4 & 20 & 10.5 & & - & - & - & - & \\
\hline $\begin{array}{l}\text { Mysis sp. } \\
\text { AMPHIPODA }\end{array}$ & 14.3 & 2.8 & 2.0 & 4.7 & & 50 & 40 & 15.7 & 26.9 & \\
\hline Gammarus gammarus & 7.2 & 2.8 & 40 & 16 & & 25 & 6.7 & 6.3 & 10.1 & \\
\hline Total Arthropoda & 28.6 & 7 & 62 & 31.2 & 3 & 75 & 46.7 & 24 & 37 & 2 \\
\hline \multicolumn{11}{|l|}{ MOLLUSCA } \\
\hline \multicolumn{11}{|l|}{ BIVALVIA } \\
\hline Flexopecten flexuosus & 50.3 & 42.4 & 6.1 & 18.4 & & 25 & 6.7 & 6.3 & 10.1 & \\
\hline Unidentified Bivalvia & 21.1 & 21.5 & 3.2 & 10.1 & & - & - & - & - & \\
\hline GASTEROPODA & & & & & & & & & & \\
\hline Turritella communis & 21.4 & 6.9 & 1.0 & 4.3 & & - & - & - & - & \\
\hline SCAPHOPODA & & & & & & & & & & \\
\hline Antalis dentalis & 14.3 & 2.6 & 0.4 & 2.1 & & - & - & - & - & \\
\hline Total Mollusca & 107.1 & 71.7 & 10.7 & 35.9 & 1 & 25 & 6.7 & 6.3 & 10.1 & 3 \\
\hline \multicolumn{11}{|l|}{ ANNELIDA } \\
\hline Nereis sp. & 18.2 & 2.5 & 20.9 & 14.4 & & 13.8 & 7.7 & 23.9 & 16 & \\
\hline Sabellidae & 10.4 & 1.1 & 8.2 & 10 & & 5.5 & 6.5 & 29.6 & 11.6 & \\
\hline Serpulidae & 4.7 & 2.3 & 11.5 & 8 & & 5.7 & 5.8 & 22 & 14 & \\
\hline Total Annelida & 33.3 & 5.9 & 40.6 & 32.4 & 2 & 25 & 20 & 75.5 & 41.6 & 1 \\
\hline
\end{tabular}


TABle 11. - Prey identified in the stomach contents of $P$. acarne adults in relation to season. $F O$, frequency of occurrence; $N$, numerical percentage; $P$, point percentage; $M F I$, Main Food Index; R, classification of prey.

\begin{tabular}{|c|c|c|c|c|c|c|c|c|c|c|}
\hline Taxa & $\begin{array}{l}\text { Summer } \\
F O(\%)\end{array}$ & $N(\%)$ & $P(\%)$ & $M F I$ & $\mathrm{R}$ & $\begin{array}{l}\text { Winter } \\
F O(\%)\end{array}$ & $N(\%)$ & $P(\%)$ & $M F I$ & $\mathrm{R}$ \\
\hline \multicolumn{11}{|l|}{$\begin{array}{l}\text { ARTHROPODA } \\
\text { CRUSTACEA DECAPODA }\end{array}$} \\
\hline Crangon crangon & 8.3 & 2.5 & 4.9 & 12.3 & & 7.9 & 6.5 & 51 & 137 & \\
\hline Ebalia sp. & 14.4 & 6.8 & 3.7 & 10.1 & & 16.3 & 6.8 & 14.9 & 12.8 & \\
\hline Liocarcinus arcuatus & 10.1 & 5.5 & 9 & 4.4 & & 13.9 & 3.3 & 6.6 & 8.1 & \\
\hline Pagurus sp. & 12.5 & 6.3 & 2 & 8.1 & & 11 & 3.8 & 2.9 & 4.6 & \\
\hline MYSIDA & & & & & & & & & & \\
\hline $\begin{array}{l}\text { Mysis sp. } \\
\text { ISOPODA }\end{array}$ & 6.6 & 5.9 & 7.8 & 3.2 & & 3.3 & 5.2 & 8.3 & 2.1 & \\
\hline $\begin{array}{l}\text { ISOPODA } \\
\text { Sphaeroma serratum }\end{array}$ & 32 & 08 & 63 & & & & & & & \\
\hline $\begin{array}{l}\text { Sphaeroma serratum } \\
\text { CUMACEA }\end{array}$ & 3.2 & 2.8 & 0.3 & 2 & & 12.1 & 8.8 & 1.7 & 6.2 & \\
\hline OSTRACODA & & & & & & & & & & \\
\hline Gammarus gammarus & 4 & 2 & 4.4 & 0.5 & & 3 & 0.9 & 6.1 & 0.7 & \\
\hline Total Arthropoda & 67.4 & 34.4 & 42.6 & 42.5 & (1) & 73.4 & 50.6 & 49.5 & 52.5 & (1) \\
\hline \multicolumn{11}{|l|}{$\begin{array}{l}\text { MOLLUSCA } \\
\text { BIVALVIA }\end{array}$} \\
\hline Flexopecten flexиоsus & 7.8 & 4 & 0.5 & 2.5 & & - & - & - & - & \\
\hline Unidentified & 16.2 & 5.5 & 0.3 & 2.5 & & 0.8 & 0.4 & 0.05 & 0.1 & \\
\hline \multicolumn{11}{|l|}{ GASTEROPODA } \\
\hline Phyllonotus trunculus & 8.2 & 3.4 & 0.1 & 1.5 & & - & - & - & - & \\
\hline Calliostoma granulatum & 3.2 & 4.2 & 0.7 & 1.5 & & 3.1 & 1.2 & 0.08 & 0.1 & \\
\hline Cerithium alucastrum & 2.1 & 8 & 0.6 & 2.6 & & - & - & - & - & \\
\hline Lunatia sp. & 2.6 & 4.1 & 0.6 & 1.8 & & - & - & - & - & \\
\hline Turritella communis & 6.1 & 3 & 0.7 & 1.2 & & 3.5 & 1.8 & 0.2 & 0.5 & \\
\hline Caecum trachea & 1.2 & 0.6 & 0.3 & 0.3 & & - & - & - & - & \\
\hline Philine catena & - & - & - & - & & 1.5 & 0.6 & 0.1 & 0.1 & \\
\hline Rissoa sp. & 3.7 & 0.8 & 0.4 & 1.2 & & 2.4 & 0.8 & 0.2 & 0.2 & \\
\hline Retusa sp. & 5.2 & 1.6 & 0.6 & 1.2 & & 2.4 & 0.7 & 0.1 & 0.2 & \\
\hline SCAPHOPODA & & & & & & & & & & \\
\hline Antalis dentalis & 9.2 & 2.2 & 1.2 & 2 & & 4.5 & 1.9 & 0.1 & 1.4 & \\
\hline $\begin{array}{l}\text { CEPHALOPODA } \\
\text { Sepia officinalis }\end{array}$ & & & & & & 1 & 05 & 006 & 08 & \\
\hline $\begin{array}{l}\text { Sepia officinalis } \\
\text { Total Mollusca }\end{array}$ & 64.5 & 37.4 & 6 & 18.9 & (3) & 19.2 & 8 & 0.8 & 3.4 & (5) \\
\hline \multicolumn{11}{|l|}{ ECHINODERMATA } \\
\hline Paracentrotus lividus & 6 & 2 & 8.9 & 5.4 & & 2.4 & 1.8 & 6.8 & 4.3 & \\
\hline Neolampas rostellata & 12 & 6.3 & 12.5 & 9.2 & & 6.5 & 1.5 & 8.2 & 6.1 & \\
\hline Amphipholis squamata & 8 & 3 & 10 & 8.6 & & 10.3 & 3.3 & 10.1 & 5.4 & \\
\hline Total Echinodermata & 26 & 11.3 & 31.4 & 23.2 & (2) & 19.2 & 6.58 & 25.1 & 15.8 & (2) \\
\hline \multicolumn{11}{|l|}{ ANNELIDA } \\
\hline Nereis sp. & - & - & - & - & & 5.7 & 4.4 & 11.2 & 5.9 & \\
\hline Sabellidae & - & - & - & - & & 4.3 & 2.3 & 7.3 & 4.7 & \\
\hline Serpulidae & 13 & 3.3 & 19.3 & 11.7 & & - & - & - & - & \\
\hline Total Annelida & 13 & 3.3 & 19.3 & 11.7 & (4) & 10 & 6.7 & 18.5 & 10.6 & (3) \\
\hline BACILLARIOPHYTA & - & - & - & - & & 1.4 & 0.4 & 0.04 & 0.2 & $(\mathbf{9 . 5})$ \\
\hline BRYOZOA & - & - & - & - & & 1.4 & 0.4 & 0.04 & 0.2 & (9.5) \\
\hline FORAMINIFERA & 10.6 & 3.5 & 0.2 & 1.1 & (6) & 20 & 12.1 & 0.6 & 3.23 & (6) \\
\hline NEMATHELMINTHA & 2.7 & 1.4 & 0.1 & 0.5 & (7) & 5.8 & 2.3 & 0.2 & 1 & (8) \\
\hline TELEOSTEI & - & - & - & - & & 3.4 & 2.3 & 13.6 & 5.6 & (4) \\
\hline PHYTOPHYTA & 8.8 & 2.8 & 0.3 & 1.2 & (5) & 13.7 & 4.3 & 0.4 & 1.9 & (7) \\
\hline
\end{tabular}

TABLE 12. - Statistical variations of the diet of $P$. acarne adults in relation to season. $\rho$, Spearman coefficient of correlation; $t_{\text {obs }}$, Student test values; (+), homogeneous diet; (-), heterogeneous diet.

\begin{tabular}{lccc}
\hline Seasons & $\rho$ & $\mathrm{t}_{\mathrm{obs}}$ & Significance $(\mathrm{p}=0.01)$ \\
\hline Summer-Autumn & 0.936 & 3.246 & + \\
Autumn-Winter & 0.6 & 1.301 & - \\
Winter-Spring & 0.463 & 1.172 & - \\
Spring-Summer & 0.765 & 2.217 & + \\
& & & + \\
\hline
\end{tabular}

\section{DISCUSSION}

The vacuity index of all the specimens together did not show any significant seasonal variation; however, the $V I$ of females was lower than that of males in autumn. This period corresponds to the gonad maturation of the axillary seabream (Mokrani et al., 2007). Females had a higher feeding intensity than males, as they need to consume more energy for the maturation of their gonads. The high vacuity index values revealed 
low feeding intensity in both sexes during spawning and post-spawning periods (i.e. winter-spring). This may be related to the lower availability of prey mainly in winter, which become less active and are closer to the bottom due to the low water temperature in the Gulf of Tunis $\left(8-13^{\circ} \mathrm{C}\right)$ (Daly Yahia, 1998), and are therefore less exposed to predation. In general, the low feeding intensity observed in this study may be due to the fishing gear and/or to the feeding behaviour of fish at the moment of capture, as observed for the Pleuronectiforms caught by static gears (Verheijen and De Groot, 1979). In the Gulf of Tunis, the axillary seabream was captured at night, which is when the fish are most active generally because they are feeding. The individuals caught were hauled onboard the following morning. Hence, some of them may have remained several hours in the net, and their capture may have occurred before the ingestion of prey or after digestion. As a result, many specimens would have had an empty stomach at the moment they were collected.

According to the data obtained, Arthropoda, Mollusca and Echinodermata were the most common prey in the diet of the axillary seabream living in the Gulf of Tunis. Annelida were secondary prey. Other prey, such as, Foraminifera, Bacillariophyta, Bryozoa, Nemathelmintha and Teleostei were of minor importance. This carnivorous type of diet conforms to those of Sparidae species belonging to the Diplodus genus reported by several authors (Rosecchi, 1987; Gonçalves and Erzini, 1998; Pallaoro et al., 2006; Derbal et al., 2007) and of other species of the Pagellus genus in particular (Larrañeta, 1964; Rijavec and Županović, 1965; Ardizzone and Messina, 1983; Rosecchi, 1983; Ghorbel, 1996).

The axillary seabream, is considered to be an euryphagous species, as it searches for food in the endofauna and crawling fauna (Mollusca, Annelida, Echinodermata). Our results agree with those of Collingnon and Aloncle (1960), who reported the presence of a large number of prey from the endofauna (Bivalvia and Annelida), fish and crustaceans in the stomachs of the axillary seabream from the Atlantic coasts of Morocco. The presence of Echinodermata in the diet of this species was also reported in the eastern Mediterranean along the Egyptian coasts (Rizkalla, 1999) and in the Atlantic, along the northwestern coasts of Africa (Phân and Kompowski, 1972) and around Azores (Morato et al., 2001). The low contribution of fish to the diet of $P$. acarne from the Gulf of Tunis contrasts with the information reported for the same species in the Atlantic, where the frequency of occurrence reached $76.3 \%$ in spring (Morato et al., 2001). The scarcity of fish in the diet of $P$. acarne observed in this study may be due to the overexploitation of local fish, which might have led to changes in the existing trophic chain at different levels. The axillary seabream probably uses its thick lips to move the sediment around in order to search for prey moving close to or inside the substratum . Unlike sparids, $P$. acarne does not have sharp anterior teeth. Prey is probably sucked up and then ground with the molars or swallowed whole. Indeed, Linde et al. (2000) showed a strong correlation between the diet and the shape of the premaxillary in sparids, but not between the diet and teeth.

The feeding behaviour of $P$. acarne was different between sexes. It showed seasonal variations, mainly in females, which could be related to the reproductive cycle. In fact, prey ingestion is adapted to the energetic needs of the fish throughout the year. In contrast, seasonal variations in males were observed only during maturation of their gonads and the spawning period (autumn-winter). Considering the liver as the storage organ of the energetic reserves, our findings are in agreement with the monthly changes in the hepatosomatic index of P. acarne, which are larger in females than in males (Mokrani et al., 2006).

The diet of juveniles was less diversified than that of adults. In summer and in winter, Crustacea and Echinodermata (i.e. prey with hard carapaces and tests) were absent from the diet of juveniles. Their diet is dominated by small organisms, such as Amphipoda (Gammarus gammarus), Mysida (Mysis sp) and bivalve juveniles, which indicates an increase in prey size in relation to fish size. This difference in feeding behaviour between juveniles and adults is also known in other sparids, such as Diplodus sargus (Rosecchi, 1987), D. vulgaris (Pallaoro et al., 2006) and D. annularis (Derbal et al., 2007). In adults, the larger opening of the mouth and the bony structure of the maxillaries, premaxillaries and mandibles, which makes them stronger, could allow the capture and ingestion of bigger and harder prey.

In conclusion, in the Gulf of Tunis $P$. acarne is a carnivorous and euryphagous predator. This species feeds on benthic organisms such as Arthropoda, Mollusca and Echinodermata. However, sex-related seasonal variations and diet differences between juveniles and adults were observed. 


\section{ACKNOWLEDGEMENTS}

The authors wish to acknowledge the suggestions and comments of two anonymous reviewers, which helped to improve the quality of the manuscript.

\section{REFERENCES}

Abellán, A. and B. Basurco. - 1999. Marine finfish species diversification: current situation and prospects in Mediterranean aquaculture. Cah. Opt. Médit., Série B. Etudes et Recherches, 24: $1-139$.

Andaloro, F. - 1983a. Résumé des paramètres biologiques sur Pagellus acarne de la mer Tyrrhénéenne méridionale et de la mer Ionienne septentrionale. FAO Fish. Rep., 266: 89-92.

Andaloro, F. - 1983b. About the catch, the diet, the reproduction, the size and distribution of Pagellus acarne (Risso, 1826) in the Strait of Messina Area. Rapp. Comm. Int. Mer Médit., 28 (5): 33-37.

Andaloro, F. and A. Rinaldi. - 1998. Fish biodiversity change in Mediterranean Sea as tropicalisation phenomenon indicator. In: E.G. D'Angelo and C. Zanolla (eds.), Indicators for Assessing Desertification in the Mediterranean, pp. 201-206. A.N.P.A., Rome.

Arculeo, M., S. Bruslé-Sicard, A. Potoschi and S. Riggio. - 2000. Investigations on gonadal maturation in Pagellus acarne (Piscies, Sparidae) in the Strait of Messina (Sicily). Ital. J. Zool., 67 (4): 333-337.

Ardizzone, G.D. and A. Messina. - 1983. Feeding habits of Pagellus erythrinus (L.) (Pisces, Sparidae) from the middle Tyrrhenian Sea. Rapp. Comm. Int. Mer Médit., 28 (5): 39-42.

Berg, J. - 1979. Discussion of methods of investigating the food of fishes, with reference to a preleminary study of the food of Gobiusculus flavescens (Gobiidae). Mar. Biol., 50: 263-273.

Bonnet, M. - 1969. Les sparidés des côtes nord-ouest africaines. Rev. Trav, Inst. Pêch. Marit, 33 (1): 97-116.

Bouchereau, J.L., C. Marques, P. Pereira, O. Guelorget and Y. Vergne. - 2006. Trophic characterization of the Prévost lagoon (Mediterranean Sea) by the feeding habits of the European eel Anguilla anguilla. Cah. Biol. Mar., 47: 133-142.

Bouhlel, M. - 1978. Le stock de poissons du plateau continental exploité à l'aide du chalut et des engins côtiers. Estimation. Prospection des fonds. Réglementation de la pêche. Rapp. doc. Inst. natn. scient. Tech. Océanogr. Pêche Salambô, (2) 1978 3-26.

Bradaï, M.N. - 2000. Diversité du peuplement ichtyque et contribution à la connaissance des sparidés du golfe de Gabès. Doctorat d'Etat, Univ. Sfax.

Campos, A. and P. Fonseca. - 2003. Selectivity of diamond and square mesh cod ends for horse mackerel (Trachurus trachurus), European hake (Merluccius merluccius) and axillary seabream (Pagellus acarne) in the shallow groundfish assemblage off the south-west coast of Portugal. Sci. Mar., 67(2): 249-260.

Coelho, R., L. Bentos, C. Correira, J.M.S. Gonçalves, P. Monteiro, J. Ribeiro, P.G. Lino and K. Erzini. - 2005. Age, growth and reproduction of the axillary seabream, Pagellus acarne (Risso, 1826) from the south coast of Portugal. Thalassas, 21(1): 79-84.

Collingnon, J. and H. Aloncle. - 1960. Le régime alimentaire de quelques poissons benthiques des côtes marocaines. Bull. Inst. Pêch. Marit. Maroc, 5: 1-13.

Daly Yahia, M.N. - 1998. Dynamique saisonnière du Zooplancton de la baie de Tunis (Systématique, écologie numérique et biogéographie méditerranéenne). Ph.D. thesis, Univ. Tunis.

D'Ancona, U. - 1949. Ermafroditsmo ed intersessualità nei Teleostei. Experientia, 5: 381-389.

Derbal, F., S. Nouacer and M.H. Kara. - 2007. Composition et variations du régime alimentaire du sparillon Diplodus annularis (Sparidae) du golfe d'Annaba (Est de l'Algérie). Cybium, 31(4): 443-450.

Domanevskaya, M.V. and F.A. Patokina. - 1984. Feeding of the large-eyed dogteeth, Dentex macrophthalmus, and Spanish bream, Pagellus acarne, from Central Eastern Atlantic Ocean. J. Ichthyol., 24: 107-112.

Dominguez, J. - 2000. Biologia pesquera del besugo Pagellus acarne (Risso, 1826) del mar de Alborán. Ph.D. thesis, Univ. Malaga.

Falissard, B. - 2005. Comprendre et utiliser les statistiques dans les sciences de la Vie (3ème édit.). Masson, Paris.

Fischer, W., M. Schneider and M.L. Bauchot. - 1987. Méditerranée et mer Noire. Zone de pêche 37, révision 1. Fiches $\mathrm{FAO}$ d'Identification des Espèces pour les Besoins de la Pêche. Vol. I, Végétaux et invertébrés. FAO, Rome.

Ghorbel, M. - 1996. Le pageot commun Pagellus erythrinus (Poisson, Sparidae) écobiologie et état d'exploitation dans le golfe de Gabès. Ph.D. thesis, Univ. Tunis.

Goncalves, J.M.S. and K. Erzini. - 1998. Feeding habits of the twobanded sea bream (Diplodus vulgaris) and the black sea bream (Spondyliosoma cantharus) (Sparidae) from the south-west coast. Cybium, 22(3): 245-254.

Greco, S., L. Genovese and V. Micale. - 1995. Growth, gonadal histology and liver lipid composition in Pagellus acarne. Cah. Opt. Médit., 16: 89-102.

Hureau, J.C. - 1970. Biologie comparée de quelques poissons antarctiques (Nototheniidae). Bull. Inst. Océanogr. Monaco, (1391): 1-244.

Hynes, H.B.N. - 1950. The food of freshwater sticklebacks (Gasterosteus aculeatus and Pygosteus pungittius) with a review of methods used in studies of the food of fishes. J. Anim. Ecol., 19: $36-58$

Larrañeta, M.G. - 1964. Sobre la biologia de Pagellus erythrinus L., especialmente de las coasts de Castellón. Inv. Pesq., 27: 121-146.

Linde, M., M. Palmer and J. Gomez-Zruta. - 2000. Differencial correlates of diet and phylogeny on the shape of the premaxilla and anterior tooth in sparid fishes (Perciformes: sparidae). J. Evol. Biol., 17: 941-952.

Lissia Frau, A.L. - 1968. Le manifestazioni della sessualità degli Sparidi. Studi Sassaresi, 4: 243-261.

Lubet, P. and A. Azouz. - 1969. Etude des fonds chalutables du golfe de Tunis. Bull. Inst. natn. Scient. Tech. Océanogr. Pêche Salammbô, 1 (3): 87-112

Mokrani, E., R. Fehri-Bedoui and O.K. Ben Hassine - 2007. Sexratio et reproduction de Pagellus acarne (Téléostéen, Sparidae) du golfe de Tunis. Actes des VIIIémes Journées tunisiennes des Sciences de la Mer. Bull. Inst. natn. Scient. Tech. Océanogr. Pêche Salammbô, 11: 96-100.

Morato, T., E. Solà, M.P. Grós and G. Menezes. - 2001. Feeding habits of two congener species of seabreams, Pagellus bogaraveo and Pagellus acarne, off Azores (northeastern Atlantic) during spring of 1996 and 1997. Bull. Mar. Sci., 69(3): 1073-1087.

Mytilineou, Ch. - 2000. Preliminary results on the reproductive cycle of Pagellus acarne (Risso 1826) in the Greek waters. In: Proceedings of the $6^{\text {th }}$ International Symposium on the Reproductive Physiology of Fish, 6: 140-140. Univ. Bergen.

Pajuelo, J.G. and J.M. Lorenzo. - 2000. Reproduction, age, growth and mortality of axillary seabream, Pagellus acarne (Sparidae), from the Canarian Archipelago. J. Appl. Ichthyol., 16 (2): 41-47.

Pallaoro, A., M. Šantić and I. Jardas. - 2006. Feeding habits of the common two-banded sea bream, Diplodus vulgaris (Sparidae), in the eastern Adriatic Sea. Cybium, 30(1): 1-96.

Pasquaud, S., M. Girardin and P. Elie. - 2004. Etude du régime alimentaire des gobies du genre Pomatochistus (P. microps et P. minutus) dans la Gironde (France). Cybium, 28(1): 99-106.

Petrakis, G. and K.I. Stergiou. - 1996. Gill net selectivity for four fish species (Mullus barbatus, Pagellus erythrinus, Pagellus acarne and Spicara flexuosa) in Greek waters. Fish. Res., 27(13): $17-27$.

Phân, Lê-T. and A. Kompowski. - 1972. The bronze bream Pagellus acarne (Risso) from North west African region. Act. Ichthyol. Piscat. 2(1): 3-18.

Riedel, R. - 1983. Fauna und flora des Midttelmeeres. Ed. Verlag Paul Parey.

Rijavec, L. and S. Županović. - 1965. A contribution to the knowledge of biology of Pagellus erythrinus L. in the middle Adriatic. Rapp. Comm. Int. Mer Médit., 18(2): 195-200.

Rizkalla, S. I., W.F. Wadie, A.S. El-Zahaby and S.S. El-Serafy. - 1999. Feeding habits of Sea breams (genus Pagellus) in the 
Egyptian Mediterranean waters. J. King Abdulaziz Univ. Press Mar. Sci., 10: 125-140.

Rosecchi, E. - 1983. Régime alimentaire du pageot, Pagellus erythrinus Linné, 1758 (Pisces, Sparidae) dans le golfe du Lion. Cybium, 7(3): 17-29.

Rosecchi, E. - 1987. L'alimentation de Diplodus annularis, Diplodus sargus, Diplodus vulgaris et Sparus aurata (Pisces, Sparidae) dans le golfe du Lion et les lagunes littorales. Rev. Trav. Inst. Pêch. Marit., 49(3-4): 125-141.

Santos, M.N., C.C. Monteiro and K. Erzini. - 1995. Aspects of the biology and gillnet selectivity of the axillary seabream (Pagellus acarne, Risso) and common pandora (Pagellus erythrinus, Linnaeus) from the Algarve (south Portugal). Fish. Res., 23(34): 223-236.

Spedicato, M.T., S. Greco, K. Sophronidis, G. Lembo, D. Giordano and A. Argyri. - 2002. Geographical distribution, abundance and some population characteristics of the species of the genus Pagellus (Osteichthyes: perciformes) in different areas of the Mediterranean. Sci. Mar., 66(Suppl. 2): 65-85.
Tokaç, A., A. Lök, Z. Tosunoglu, C. Metin and R.S.T. Ferro. - 1998. Cod-end selectivities of a modified bottom trawl for three fish species in the Aegean Sea. Fish. Res., 39(1): 17-31.

Verheijen, F.T. and D.J. De Groot. - 1979. Diurnal activity pattern of Plaice and Flounder (Pleuronectidae) in aquaria. Nether. J. Sea Res., 3(3): 383-390.

Zander, C.D. 1982. - Feeding ecology of littoral Gobiid and Blennioid of the Banyuls area (Mediterranean Sea) - I. Main food and trophic dimension of niche and ecotope. Vie Milieu, 32: $1-10$.

Zerouali-Khodja, F. and N. Amalou. - 2005. Etude anatomique et histologique des gonades d'un poisson hermaphrodite, Pagellus acarne (Risso, 1829) des côtes algéroises. Bull. Soc. Zool. Fr., 130(1): 81-93.

Scient. ed.: A. Sabatés.

Received June 17, 2008. Accepted January 26, 2009.

Published online July 6, 2009. 